\title{
Postoperative catheterization after anterior colporrhaphy: 2 versus 5 days. A multicentre randomized controlled trial
}

\author{
Mirjam Weemhoff • Martine M. L. H. Wassen • \\ Laura Korsten • Jan Serroyen • \\ Paul H. N. M. Kampschöer • Frans J. M. E. Roumen
}

Received: 4 March 2010 / Accepted: 5 October 2010 / Published online: 20 October 2010

(C) The Author(s) 2010. This article is published with open access at Springerlink.com

\begin{abstract}
Introduction and hypothesis The aim of this study was to compare the number of temporary catheter replacements and urinary tract infections after indwelling catheterization for 2 versus 5 days following an anterior colporrhaphy.

Methods Two hundred forty-six patients were randomly assigned to 2 or 5 days of indwelling catheterization. Outcome measures were temporary catheter replacements because of post-voiding residual $>200 \mathrm{~mL}$ after removal of the indwelling catheter, urinary tract infections, and hospital stay. All patients were analyzed according to the intention to treat principle.

Results Compared to the 5-day protocol group, in the 2-day protocol group more patients needed temporary catheter replacement $(9 \%$ versus $28 \%$, odds ratio (OR) 4.0 , confidence interval (CI) 1.9-8.3, $p<0.01$ ), whereas less patients had a urinary tract infection $(37 \%$ versus $22 \%$, OR 0.5 , CI $0.3-0.9$, $p=0.02$ ) and median hospital stay was lower.
\end{abstract}

\footnotetext{
M. Weemhoff $(\bowtie) \cdot$ M. M. L. H. Wassen • L. Korsten Department of Obstetrics \& Gynecology, GROW-School for Oncology and Developmental Biology, Maastricht University Medical Centre,

P.O.Box 5800, 6202 AZ Maastricht, The Netherlands

e-mail: m.weemhoff@mumc.nl

J. Serroyen

Department of Methodology and Statistics, Maastricht University, Maastricht, The Netherlands

P. H. N. M. Kampschöer • F. J. M. E. Roumen Department of Obstetrics \& Gynecology, Atrium Medical Centre, Heerlen, The Netherlands
}

Conclusions Removal of an indwelling catheter after 2 versus 5 days following anterior colporrhaphy is associated with more temporary catheter replacements, but less urinary tract infections and a shorter hospital stay.

Keywords Cystocele · Anterior colporrhaphy Indwelling catheterization - Urinary retention - Temporary catheter replacement $\cdot$ Urinary tract infection

\section{Introduction}

A nationwide questionnaire-based survey in 99 hospitals in The Netherlands has shown a mean duration of catheterization of 3.7 days (1-7 days) following an anterior colporrhaphy [1]. Drainage of urine is being performed to reduce the risk of retention by postoperative edema of the bladder. Overfilling of the bladder might have a negative impact on the surgical outcome [2]. Only three studies have been published on the effects of the duration of postoperative indwelling catheterization following an anterior colporrhaphy. The first study in 106 women did not find a significant difference in incidence of urinary retention between catheter removal on the first (24\%) and third day (31\%) postoperatively [3]. It was concluded that the indwelling catheter can be removed on the first day postoperatively. A subsequent underpowered study in 50 patients did not find a difference in incidence of urine retention between the first and third postoperative day either, but showed considerably lower retention percentages in both groups (8\%) [4]. In contrast, a randomized controlled trial in The Netherlands in 100 patients showed 
$40 \%$ of patients having a post-voiding residual of more than $200 \mathrm{~mL}$ after removal of the catheter on the first postoperative day versus $9 \%$ of patients having retention after removal of the catheter on the fifth postoperative day [2]. We concluded that removal of the indwelling catheter on the first day postoperatively is too soon. Based on these conclusions, we hypothesized that 2 days of indwelling catheterization postoperatively after an anterior colporrhaphy is more appropriate than 5 days, which is most commonly applied in The Netherlands.

Bladder catheterization, however, increases the risk of urinary tract infections [5]. After removal of the catheter on the first day postoperatively, only $4 \%$ of patients had infections, compared to $20 \%$ when the catheter had been removed on the third day and $40 \%$ after removal of the catheter on the fifth day $[2,4]$.

In a retrospective study, high-grade cystocele, higher intra-operative blood loss, Kelly plication, and levator plication were identified by multivariable analysis as independent risk factors for short-term urinary retention after vaginal prolapse surgery [6].

The aim of the present study was to determine in a randomized controlled trial the incidence of bladder retention and urinary tract infections after postoperative indwelling catheterization for 2 days versus indwelling catheterization for 5 days.

\section{Methods}

Between January 2006 and September 2008, a randomized controlled trial was performed in three different hospitals in patients undergoing an anterior colporrhaphy comparing postoperative temporary indwelling catheterization for 2 days versus indwelling catheterization for 5 days. The primary outcome measure was the number of temporary catheter replacements after the first removal of the catheter postoperatively. A catheter was temporarily replaced in case the residual volume after micturition was more than $200 \mathrm{~mL}$. Despite the definition of the International Continence Society for bladder retention, which is one third of the bladder volume, the cut-off value of $200 \mathrm{~mL}$ was chosen to make the results of the study comparable to those of another study [2]. Secondary outcome measures were the rate of urinary tract infections and the length of the hospital stay. Furthermore, the factors that appeared to be associated with postvoiding residuals more than $200 \mathrm{~mL}$ after removal of the catheter in univariable regression were assessed by multivariate logistic regression to find out if they were independent risk factors for temporary catheter replacements. The assessed factors in univariable analysis were age, BMI, history of obstructive micturition or recurrent urinary tract infections, advanced stage of prolapse preoperatively, used operation techniques, concomitant surgery, mean amount of blood loss, urinary tract infections, and the protocol for catheterization used.

All patients with a cystocele undergoing an anterior colporrhaphy were eligible. As the POP-Q classification was not generally introduced in daily practice at the study start, prolapse was staged at the outpatient clinic according to the Baden-Walker classification. Stage I was a cystocele, rectocele, or descensus uteri into the first half of the vagina, in stage II the prolapse protruded into the distal half of the vagina, in stage III the prolapsed tissue bulged out of the vagina, and stage IV was a total protrusion of the bladder, rectum, or uterus.

Doctors were encouraged to perform the anterior repair as they were used to. Combinations with other vaginal operations like hysterectomy, sacrospinal fixation, or posterior colporrhaphy were allowed. Excluded were women who were performing self-catheterization because of voiding dysfunctions preoperatively, women under 18 years of age, and those who were not able to understand informed consent because of low IQ or a language barrier. After informed consent, patients were included at the outpatient clinic at the time the operation was planned. The protocol was approved by the medical ethical committees of the three participating hospitals.

Patients were randomized to postoperative temporary indwelling catheterization for either 2 or 5 days. A randomization list was made by an independent statistician. Randomization was performed in blocks and was stratified for the different hospitals. According to the randomization list, opaque, numbered, and sealed envelopes were prepared by an independent person. At the start of the operation, urine was collected for sedimentation. After the operation was finished, the indwelling catheter was inserted; the envelope with study number was opened, and at that moment, the patient was randomized to temporary indwelling catheterization for either 2 or 5 days.

The catheter was removed in the morning of the second or fifth day postoperatively. The residual volume was measured by bladder ultrasound scan $6-8 \mathrm{~h}$ later. A residual volume after micturition of more than $200 \mathrm{~mL}$ was considered abnormal, and a new indwelling catheter was inserted. A required minimum void was not defined. The next morning, the catheter was removed, and again the post-voiding residual volume was assessed 6-8 h later. If the volume was still more than $200 \mathrm{~mL}$, a new catheter was inserted, this time for 3 days. In case the residual volume was still more than $200 \mathrm{~mL}$ after these three extra days, the patient started intermittent selfcatheterization until the residual volume after spontaneous micturition was less than $200 \mathrm{~mL}$.

All patients received prophylactic antibiotics at the beginning of the operation. Postoperative prophylactic 
antibiotics were not given routinely. After removal of the catheter, urine samples were taken for sedimentation and culture. Patients with complaints and sedimentation showing signs of urinary tract infection were given antibiotics. Signs of urine tract infection were defined as having more than 25 white blood cells per high power field, nitrate production, or more than 20 bacteria per high power field. When urinary tract infection after the removal of the catheter was confirmed by a positive culture, patients were treated with antibiotics irrespective of complaints. A culture was scored positive when the sample contained more than $10^{5}$ colony forming units per milliliter. For the outcome measure urinary tract infection, only the infections proven by a positive culture at the time of the first removal of the catheter were included. No other urinary cultures were taken on behalf of the study protocol.

All patients were given the opportunity to leave the hospital on the third day postoperatively. For patients in the 5-day protocol who requested to leave the hospital with an indwelling catheter in situ, an appointment was made on the fifth day in the outpatient clinic for ultrasound 6-8 $\mathrm{h}$ after removal of the catheter by themselves at home.

The primary outcome measure in the study was the number of patients with temporary catheter replacements after removal of the first indwelling catheter postoperatively. Based on retrospectively collected data in one of the participating hospitals, the average percentage of patients needing repeated catheterization after removal of the catheter on the fifth day after an anterior colporrhaphy was $10 \%$. To show a difference of at least $15 \%$ in the number of repeated catheterizations between a protocol of 2 versus 5 days, with a significance of 0.05 and a power of $90 \%$, it was calculated that 112 patients in each group were needed.

The study was started with the intention to include 224 patients. After 2 years of inclusion, an amendment to the study was made to perform long-term follow-up 2 years postoperatively. Therefore, the intended inclusion was increased to 250 patients, taking into account a loss to long-term follow-up of $20 \%$.

All data were collected and analyzed in SPSS 15.0 for Windows. The $t$ test, Pearson chi-square test, Fisher's exact test, and univariable and multivariable logistic regression were used in the statistical analysis of all data. All patients were analyzed according to the intention to treat principle.

\section{Results}

Between January 2006 and September 2008, 390 patients underwent an anterior colporrhaphy. Figure 1 shows the patient flow of the study. Two hundred fifty out of 390 patients (64\%) agreed to be included in the study. Three patients withdrew from the study just before the operation. One patient did not undergo the planned operation because under general anesthesia a tumor in the rectum was palpated and treatment was taken over by the surgeon. Two hundred forty-six patients were randomized after the operation. One patient, randomized to the 2-day protocol, died of a heart attack on the first postoperative day with the catheter in situ. She died before she could participate in the study. Two patients allocated to the 5-day protocol had their catheter removed on the third day because of miscommunication. The three patients were analyzed in the allocated group. In $20 \%$ of patients, the protocol was not followed and a culture of the urine was not obtained after removal of the catheter. Follow-up after 6 weeks was achieved in 245 patients.

Patient characteristics in both groups are shown in Table 1. There were no significant differences between both groups with respect to age, BMI, Baden-Walker classification of cystocele and rectocele, history of symptoms, history of surgery, pre-operative urine tract infections, concomitant surgery, mean amount of blood loss and use of levator sutures, or tobacco plication of the bladder. However, more patients had undergone Kelly sutures in the 5-day protocol group than in the 2-day protocol group.

Table 2 shows the outcome measures of the two groups. Twenty-eight percent of patients in the 2-day protocol needed temporary catheter replacement compared to $9 \%$ of patients in the 5-day protocol $(p<0.01)$. In 196 patients, urine was sent for culture after removal of the catheter. After 2 days, significantly less patients had urinary tract infections, proven by a culture with $>10^{5}$ colony forming units per milliliter, than after 5 days of catheterization $(22 \%$ versus $37 \%, p=0.02$ ). Of all patients, $67 \%$ had an uneventful postoperative period, defined as having no post-voiding residual volume more than $200 \mathrm{~mL}$ and having no urinary tract infection at time of the first catheter removal. There was no difference between the two protocols in number of patients with an uneventful postoperative period $(67 \%$ versus $66 \%, p=0.86)$.

The median duration of hospital stay was shorter in the 2-day protocol group, compared to the 5-day protocol group (median 3 versus 5 days).

To prevent one temporary indwelling catheter replacement, six patients should have their catheter left in place for 5 days instead of 2 days. Giving a patient a catheter for 5 days instead of 2 days lengthened the hospital stay by 2 days.

Table 3 shows the association between the two outcome measures: urinary tract infections and temporary catheter replacement. In the whole study population, a urinary tract infection at time of first catheter removal was associated with a post-voiding residual of more than 
Fig. 1 Patient flow

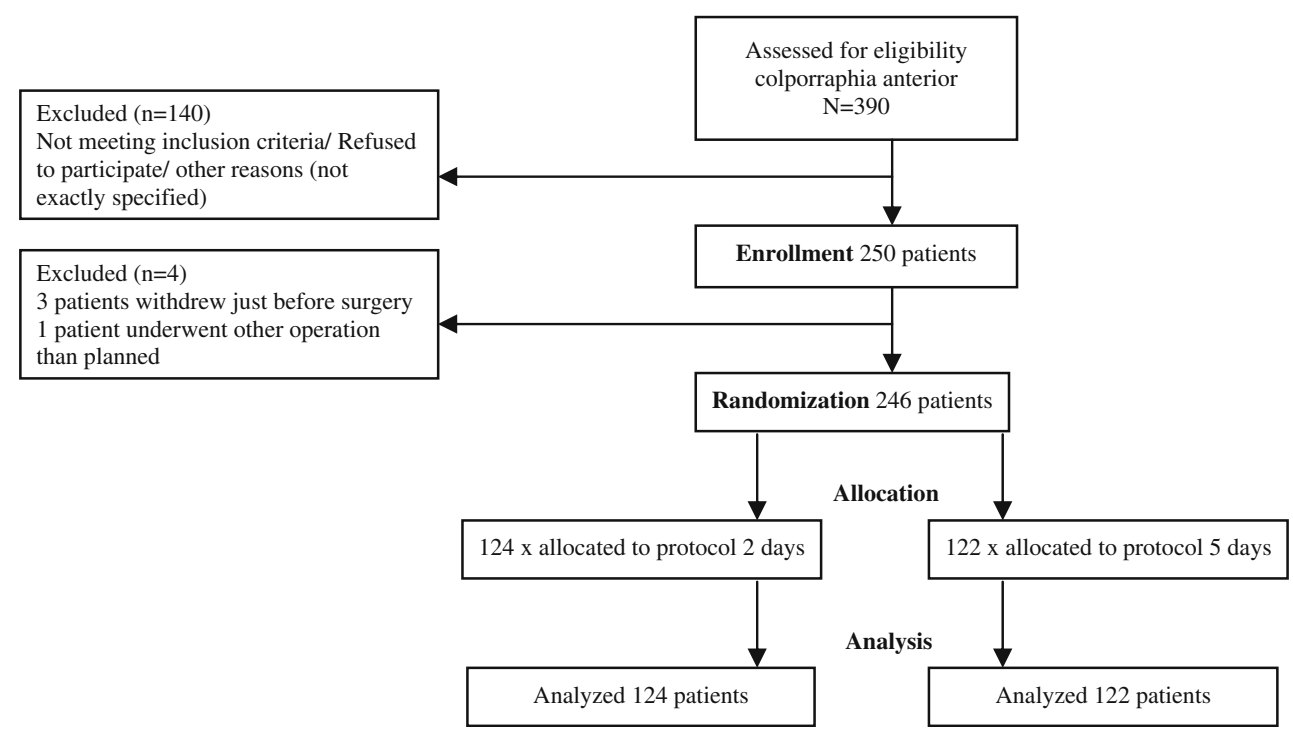

Table 1 Baseline characteristics of the study population

\begin{tabular}{|c|c|c|c|}
\hline & $\begin{array}{l}\text { Protocol } 2 \text { days catheterization } \\
(n=124)\end{array}$ & $\begin{array}{l}\text { Protocol } 5 \text { days catheterization } \\
(n=122)\end{array}$ & $p$ value \\
\hline Mean age in years & $59.9(\mathrm{SD} 10.2)$ & $60.7(\mathrm{SD} 11.1)$ & 0.57 \\
\hline Mean BMI in $\mathrm{kg} / \mathrm{m}^{2}$ & $26.6(\mathrm{SD} 3.9)$ & $26.4(\mathrm{SD} 3.9)$ & 0.64 \\
\hline \multicolumn{4}{|l|}{ History of surgery } \\
\hline History of prolapse repair & $9 \%(11)$ & $12 \%(15)$ & 0.39 \\
\hline History of hysterectomy & $20 \%(25)$ & $20 \%(24)$ & 0.90 \\
\hline History of anterior colporrhaphy & $3 \%(4)$ & $7 \%(8)$ & 0.23 \\
\hline History of posterior colporrhaphy & $5 \%(6)$ & $6 \%(7)$ & 0.76 \\
\hline \multicolumn{4}{|l|}{ History } \\
\hline Obstructive micturition & $30 \%(37)$ & $31 \%(38)$ & 0.86 \\
\hline Recurrent urinary tract infections & $8 \%(10)$ & $11 \%(13)$ & 0.50 \\
\hline Urinary tract infection in pre-operative sedimentation & $5 \%(6)$ & $6 \%(7)$ & 0.76 \\
\hline \multicolumn{4}{|l|}{ Cystocele (Baden-Walker) } \\
\hline I & $11 \%(14)$ & $11 \%(13)$ & 0.86 \\
\hline II & $35 \%(43)$ & $38 \%(47)$ & 0.56 \\
\hline III & $54 \%(67)$ & $51 \%(62)$ & 0.66 \\
\hline \multicolumn{4}{|l|}{ Rectocele (Baden-Walker) } \\
\hline 0 & $37 \%(45)$ & $37 \%(44)$ & 0.99 \\
\hline I & $34 \%(42)$ & $39 \%(46)$ & 0.50 \\
\hline II & $21 \%(25)$ & $18 \%(21)$ & 0.58 \\
\hline III & $8 \%(11)$ & $7 \%(8)$ & 0.66 \\
\hline \multicolumn{4}{|l|}{ Technique } \\
\hline Kelly sutures & $36 \%(44)$ & $47 \%(58)$ & $<0.01$ \\
\hline Levator sutures & $27 \%(33)$ & $32 \%(39)$ & 0.15 \\
\hline Tobacco sutures & $17 \%(21)$ & $18 \%(22)$ & 0.84 \\
\hline \multicolumn{4}{|l|}{ Concomitant surgery } \\
\hline Hysterectomy & $53 \%(65)$ & $55 \%(67)$ & 0.80 \\
\hline Posterior colporrhaphy & $46 \%(57)$ & $52 \%(63)$ & 0.44 \\
\hline Sacrospinal fixation & $15 \%(19)$ & $13 \%(16)$ & 0.72 \\
\hline Mean amount of blood loss in $\mathrm{ml}$ & 241 & 233 & 0.55 \\
\hline
\end{tabular}

(n) number of patients, $S D$ standard deviation 
Table 2 Outcome measures comparing the 2 and 5 days protocol

\begin{tabular}{|c|c|c|c|c|}
\hline & Protocol 2 days & Protocol 5 days & $\begin{array}{l}\text { OR }(95 \% \\
\text { CI) }\end{array}$ & $\begin{array}{l}p \\
\text { value }\end{array}$ \\
\hline Percentage of patients needing temporary catheter replacement $(n)$ & $28 \%(35 / 123)$ & $9 \%(11 / 122)$ & $4.0(1.9,8.3)$ & $<0.01$ \\
\hline $\begin{array}{l}\text { Percentage of patients with a urinary tract infection at the time of first catheter } \\
\text { removal }(n)^{\mathrm{a}}\end{array}$ & $22 \%(22 / 101)$ & $37 \%(35 / 95)$ & $0.5(0.3,0.9)$ & 0.02 \\
\hline Percentage of patients with uneventful postoperative period ${ }^{\mathrm{b}}$ & $67 \%(82 / 123)$ & $66 \%(80 / 122)$ & $1.0(0.6,1.6)$ & 0.86 \\
\hline Hospital stay Median (range) & $3.0(2.0-42.0)$ & $5.0(1.0-59.0)$ & & $<0.01$ \\
\hline
\end{tabular}

${ }^{a}$ Urinary tract infection defined as culture with $>10^{5}$ colony forming unit per milliliter

${ }^{\mathrm{b}}$ Uneventful postoperative period defined as post-voiding residual $<200 \mathrm{ml}$ and no urinary tract infection (culture $<10^{5}$ colony forming unit per milliliter) at time of first catheter removal

$200 \mathrm{~mL}$ needing catheter replacement with an odds ratio [OR] of 4.8 (95\% confidence interval [CI] $(2.3,10.2)$; $p<0.01)$. This association was stronger in the 2-day protocol group compared with the 5-day protocol group (OR 12.4; 95\% CI 4.1, 37.3; $p<0.01$ versus OR $6.0 ; 95 \%$ CI 1.1, 31.6; $p=0.03$ ).

Table 4 shows the univariable and multivariable analyses of different variables to indicate whether they form risk factors for temporary indwelling catheter replacement. The multivariable analysis focused on risk factors for catheter replacement taking into account all significant factors in the univariable analysis. A urinary tract infection at the time of the first removal of the catheter and the protocol for catheterization itself appeared to be the only significant risk factors for temporary catheter replacement.

\section{Discussion}

The reason for prolonged drainage of urine by an indwelling catheter after an anterior repair is that postoperative edema of the bladder, or innervation trauma of the bladder, can cause voiding difficulties with increasing risk of retention. Overfilling of the bladder might possibly have a negative impact on the surgical outcome [2]. The question is how long postoperative catheterization should be continued. A Cochrane review focusing on catheterization of adults undergoing a uro- genital procedure was not able to draw conclusions on whether a catheter should be inserted after an anterior repair for a shorter or longer period postoperatively to prevent repeated catheterizations [7].

In our study, $28 \%$ of patients in the 2-day protocol needed repeated temporary catheterization compared to $9 \%$ of patients in the 5 -day protocol $(p<0.01)$. These results are in line with a previous study, in which $40 \%$ of patients had retention after removing the catheter on the first postoperative day versus $9 \%$ of patients after removing the catheter on the fifth postoperative day [2]. To prevent one patient from needing repeated temporary catheterization, six patients in our study should have their catheter left in place for 5 days instead of 2 days. Giving a patient a catheter for 5 days instead of 2 days lengthened the hospital stay by 2 days, resulting in considerably higher hospital costs. This confirms findings by other investigators [7]. However, lengthening of hospital stay is probably not the result of the medical condition per se, but merely a result of the reluctance of many patients to leave the hospital with an indwelling catheter in situ and anxiety about removing the catheter by themselves.

More patients underwent Kelly sutures in the 5-day protocol group compared to the 2-day protocol group. Kelly sutures, theoretically, can obstruct the urethra causing obstructive voiding with the risk of post-voiding residual volumes. If Kelly sutures had influenced the results of the study, they would have had a negative impact on the amount of catheter replacements in the 5-day protocol group. In

Table 3 Urinary tract infections proven by a positive culture at the time of the first catheter removal

\begin{tabular}{lccc}
\hline & $\begin{array}{c}\text { Urinary tract infections in patients with } \\
\text { post-voiding residual }>200 \mathrm{ml}\end{array}$ & $\begin{array}{l}\text { Urinary tract infections in patients with } \\
\text { post-voiding residual }<200 \mathrm{ml}\end{array}$ & OR (95\% CI), $p$ value \\
\hline Protocol 2 days & $16 / 30(53 \%)$ & $6 / 71(8 \%)$ & $12.4(4.1,37.3),<0.01$ \\
Protocol 5 days & $6 / 8(75 \%)$ & $29 / 87(33 \%)$ & $6.0(1.1,31.6), 0.03$ \\
Total group & $22 / 38(58 \%)$ & $35 / 158(22 \%)$ & $4.8(2.3,10.2),<0.01$ \\
\hline
\end{tabular}


Table 4 Risk factors for temporary catheter replacement after anterior colporrhaphy

\begin{tabular}{|c|c|c|c|c|c|c|}
\hline \multirow[t]{2}{*}{ Possible risk factors } & \multicolumn{3}{|c|}{ Univariable analysis } & \multicolumn{3}{|c|}{ Multivariable analysis } \\
\hline & OR & $95 \% \mathrm{CI}$ & $p$ value & OR & $95 \% \mathrm{CI}$ & $p$ value \\
\hline Age & 1.0 & $1.0,1.0$ & 0.75 & & & \\
\hline BMI & 1.1 & $1.0,1.2$ & 0.05 & 1.1 & $1.0,1.2$ & 0.09 \\
\hline \multicolumn{7}{|l|}{ History } \\
\hline Obstructive micturition & 1.3 & $0.6,2.7$ & 0.46 & & & \\
\hline Recurrent urinary tract infections & 2.6 & $0.6,11.5$ & 0.21 & & & \\
\hline Urinary tract infection in pre-operative sedimentation & 0.78 & $0.2,3.6$ & 0.75 & & & \\
\hline Pre-operative grade III cystocele (BW) & 1.2 & $0.7,2.4$ & 0.52 & & & \\
\hline \multicolumn{7}{|l|}{ Technique } \\
\hline Kelly sutures & 0.7 & $0.3,1.3$ & 0.26 & 2.3 & $0.8,6.3$ & 0.11 \\
\hline Levator sutures & 0.8 & $0.4,1.7$ & 0.58 & & & \\
\hline Tobacco sutures & 3.4 & $1.6,7.1$ & $<0.01$ & & & \\
\hline \multicolumn{7}{|l|}{ Concomitant surgery } \\
\hline Hysterectomy & 0.9 & $0.5,1.8$ & 0.80 & & & \\
\hline Posterior colporrhaphy & 2.0 & $1.1,3.9$ & 0.04 & 1.9 & $0.8,4.7$ & 0.12 \\
\hline Mean amount of blood loss in $\mathrm{ml}$ & 1.0 & $1.0,1.0$ & 0.07 & & & \\
\hline Protocol of 2 days catheterization (compared to 5 days catheterization) & 4.0 & $1.9,8.4$ & $<0.01$ & 11.1 & $3.8,32.1$ & $<0.01$ \\
\hline Urinary tract infection at the time of the first catheter removal & 4.8 & $2.3,10.2$ & $<0.01$ & 9.1 & $3.4,23.8$ & $<0.01$ \\
\hline
\end{tabular}

contrast to others and to the theoretical concept, Kelly plication was not found an independent risk factor for repeated temporary catheterization in our study [8].

In our study, significantly less patients had urinary tract infections after 2 days than after 5 days of catheterization ( $22 \%$ versus $37 \%, p=0.02$ ). These findings are similar to previous studies, in which only $4 \%$ of patients had infections after removing the catheter on the first day postoperatively, compared to $20 \%$ on the third day and $40 \%$ on the fifth day $[2,4]$. The presence of urinary tract infections was associated with a higher number of temporary catheter replacements both in the 2-day protocol and the 5-day protocol group.

There are several weaknesses of this study that have to be acknowledged. One could criticize the choice of comparing catheterization for 2 and 5 days postoperatively. Many surgeons do remove the catheter earlier than after 5 days postoperatively. Nevertheless, in the literature, there is no clear evidence that it is better to remove a catheter shortly after pelvic floor surgery other than the subjective feeling that 5 days is long. The participating hospitals were used to give a catheter for 5 days, and referring to an average catheterization in 2003 of 3.7 days in the hospitals in The Netherlands, longer duration of catheterization was not uncommon [1].

One of the outcome measures of the study was the rate of urinary tract infections after the first catheter removal. In $20 \%$ of patients, no urine for culture was obtained after removal of the catheter. With 196 patients, the power of the study for this question is still sufficient, but it is likely that in patients with complaints, the risk of forgetting to obtain urine for culture is not that high as in patients with no complaints. It is possible that the percentage of infections was lower if cultures had been taken in all 245 patients. Because this protocol violation occurred equally in both arms (27 versus 24 patients), it causes no differences between the two arms of the study.

\section{Conclusions}

Based on the results of this study, we advise removing an indwelling catheter after an anterior repair on the second day postoperatively, followed by taking a urine culture. In case of a urinary tract infection, the risk of temporary catheter replacement is increased.

Acknowledgements We would like to thank Prof. Dr. J.L.H. Evers for reading and commenting the manuscript.

Financial support None.

Conflicts of interest None.

Open Access This article is distributed under the terms of the Creative Commons Attribution Noncommercial License which permits any noncommercial use, distribution, and reproduction in any medium, provided the original author(s) and source are credited. 


\section{References}

1. Hakvoort RA, Burger MP, Emanuels MH, Roovers JP (2009) A nationwide survey to measure practice variation of catheterization management in patients undergoing vaginal prolapse surgery. Int Urogynecol J 20:813-818

2. Hakvoort RA, Elberdink R, Vollebregt A, Ploeg vd T, Emanuel MH (2004) How long should urinary bladder catheterization be continued after vaginal prolapse surgery? A randomized controlled trial comparing short term versus long term catheterization after vaginal prolapse surgery. BJOG 111:828-830

3. Guzman S, Israel E, Puente R, Iglesias R, Rosa G, Uloa C (1994) Handling of Foley catheter regarding urinary retention syndrome following vaginal surgery. Rev Chil Obstet Ginecol 59(4):280-283

4. Alonzo-Sosa JE, Flores Contreras JT, Pardes-Canul M (1997) Method for transurethral catheterization for 1-3 days for pelvic floor relaxation in the postoperative period. Ginecol Obstet Méx 65:455-457

5. Niël-Weise BS, van den Broek PJ (2005) Antibiotic policies for short-term catheter bladder drainage in adults. Cochrane Database of Systematic Reviews 2005, Issue 3. Art. no.: CD005428

6. Hakvoort RA, Dijkgraaf MG, Burger MP, Emanuel MH, Roovers JPWR (2009) Predicting short-term urinary retention after vaginal prolapse surgery. Neurourol Urodyn 28(3):225228

7. Phipps S, Lim YN, McClinton S, Barry C, Rane A, N'Dow JMO (2006) Short term urinary catheter policies following urogenital surgery in adults. Cochrane Database of Systematic Reviews 2006, Issue 2. Art. no.: CD004374

8. Thaweekul Y, Bunyavejchevin S, Wisawasukmongchol W, Santingamkun A (2004) Long term results of anterior colporrhaphy with Kelly plication for the treatment of stress urinary incontinence. J Med Assoc Thai 87(4):357-360 